\title{
Modeling and Vibration Suppression for Telescopic Systems of Structural Members with Clearance
}

\author{
PROF. DR. DiETER ARNOLD \\ DiPL.-ING. MARTIN MITTWOLLEN \\ DIPL.-ING. FRANK SCHÖNUNG, \\ UNIVERSITÄT KARLSRUHE, INSTITUT FÜR FÖRDERTECHNIK UND LOGISTIKSYSTEME) \\ Prof. DR.-ING. JÖRG WAUER \\ DIPL.-ING. PIERRE BARTHELS \\ UNIVERSITÄT KARLSRUHE, INSTITUT FÜR TECHNISCHE MECHANIK
}

\begin{abstract}
Telescopic systems of structural members with clearance are found in many applications, e.g., mobile cranes, rack feeders, fork lifters, stacker cranes (see Figure 1). Operating these machines, undesirable vibrations may reduce the performance and increase safety problems. Therefore, this contribution has the aim to reduce these harmful vibrations. For a better understanding, the dynamic behaviour of these constructions is analysed. The main interest is the overlapping area of each two sections of the above described systems (see markings in Figure 1) which is investigated by measurements and by computations. A test rig is constructed to determine the dynamic behaviour by measuring fundamental vibrations and higher frequent oscillations, damping coefficients, special appearances and more. For an appropriate physical model, the governing boundary value problem is derived by applying Hamilton's principle and a classical discretisation procedure is used to generate a coupled system of nonlinear ordinary differential equations as the corresponding truncated mathematical model. On the basis of this model, a controller concept for preventing harmful vibrations is developed.
\end{abstract}
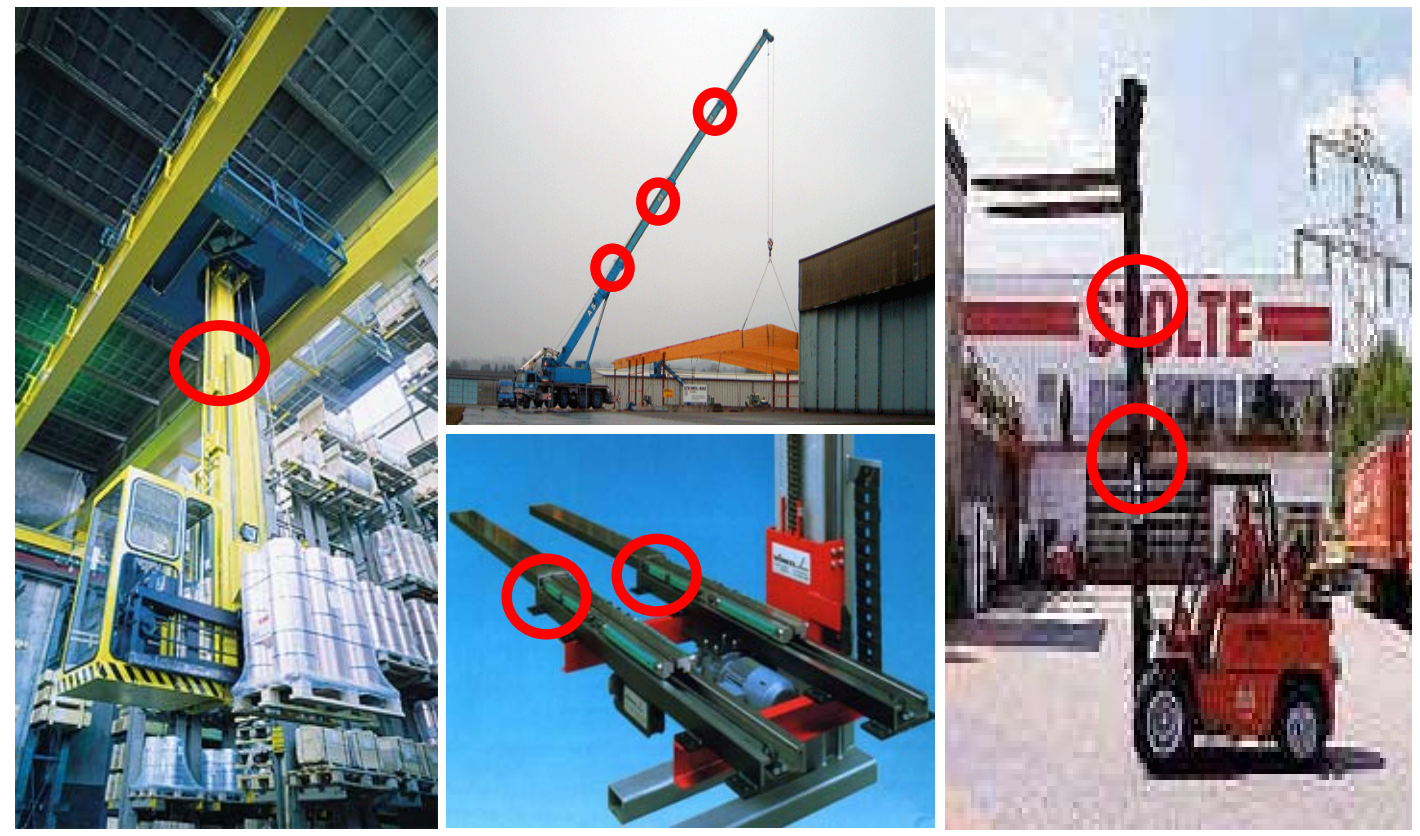

Fig.1: Graduated multi-section systems with clearance 


\section{Introduction}

Graduated multi-section systems of structural components extending and retracting inside each other are interesting technical systems. They are found, e.g., in mobile cranes, rack feeders, fork lifters, stacker cranes (see Figure 1). As the main duty of these machines is not driving around but loading and unloading goods to and from racks, trucks etc., a great number of acceleration and deceleration operations occur. Combined with the extending and retracting motion of the sections, bending vibrations of the system perpendicular to the telescopic axis occur. These vibrations lead to a significant reduction of the performance due to the required waiting time for relaxation and to safety problems so that controlled vibration suppression seems to be necessary.

The objective of the present paper is to develop a controller concept for preventing harmful vibrations. First, a system without clearance and with a fixed telescopic length which can be characterized by a time-invariant system of linear differential equations, is reduced to its dominating modes. Using this reduced model, a concept of state control via pole placement is designed which exhibits the desired effects. Introducing a so-called Luenberger observer, straightforward measurements of the motion of the telescope base and of the control variable of the actuator are sufficient to operate the controller. For real telescopic operations an adaptive controller and observer are introduced. The controller, developed for the reduced linear system model, is applied to the significantly more complicated system with clearance for studying the influence of clearance on the vibration suppression during telescopic motions.

\section{Test Rig}

As seen in Figure 1, the appearance of graduated telescopic multi-section systems with clearance can be very different. Nevertheless the main problem can be concentrated to the overlapping area of each two sections (see markings in Figure 1). Only the orientation in space is different as shown in the functional sketch of the system (Figure 2). This sketch leads on the one hand to an appropriate test rig and on the other hand to an appropriate physical model.

Main interest: overlapping area of two sections.

Difference: orientation in space.
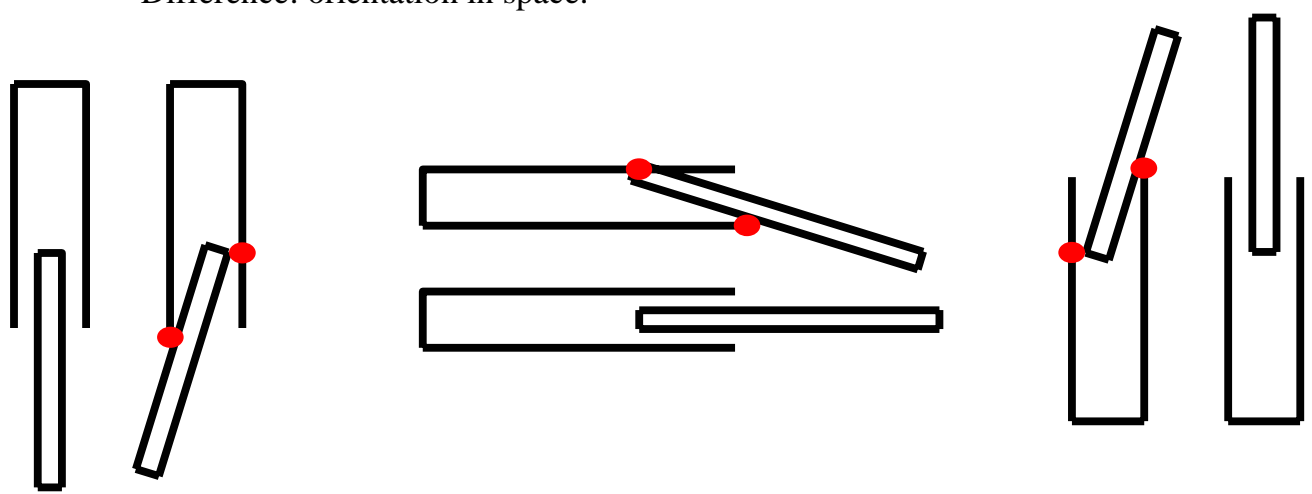

Fig. 2: Sketch of the main item - the overlapping area

The test rig, as shown in Figure 3 consists of two sections, made of slender steel-beams. The lower section is fixed to a rigid block on the ground; the upper section is connected to the lower section with screws. For choosing an assembly without clearance or with a well defined clearance, a special system of sliding bolts and bushes is used to reduce friction to a minimum and to enable free translation and rotation movement. This test rig is made of elements with a very simple geometry, because the elastic properties of these elements are well known and the measurement results are comprehensible. 


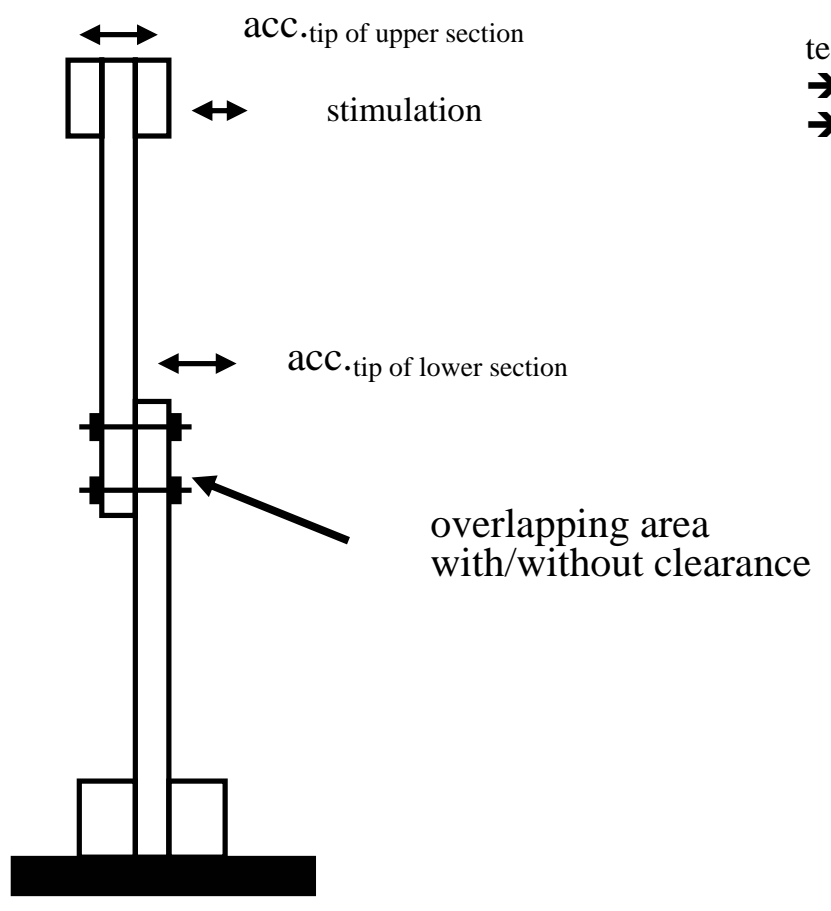

test rig with most simply geometry for:

$\rightarrow$ analysing dynamic behaviour

$\rightarrow$ analysing controller behaviour

Fig. 3: Test rig

In the following, two typical assemblies are shown in two movies. Movie 1 shows the dynamic behaviour of a two-sectional graduated system without clearance. On the left side, you can see the whole test rig, in the upper window, the overlapping area can be seen and in the lower window, the development of the vibrations can be observed, presented by the acceleration signals of the sections tips. The movie is shown in slow-motion; the fundamental frequency is $0.53 \mathrm{~Hz}$. As expected, the vibration development is absolutely harmonic.

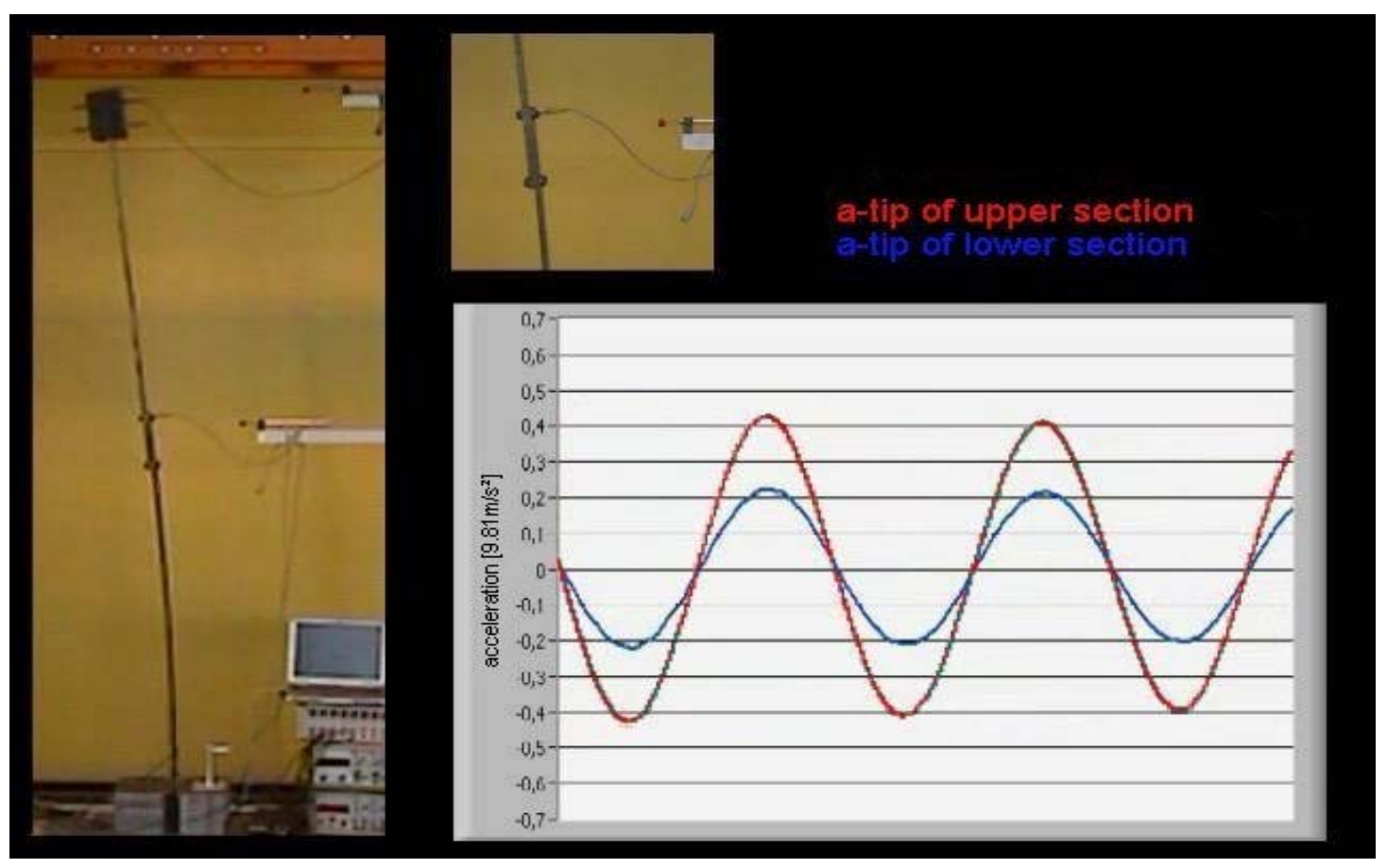

Mov. 1: System without clearance 
Movie 2 shows the dynamic behaviour of a two-sectional graduated system with clearance ( $2 \mathrm{~mm})$. On the left side, you can see the whole test rig, in the upper window, the overlapping area can be seen and in the lower window, the development of the vibrations can be observed, presented by the acceleration signals of the sections tips. The movie is shown in slow-motion; the fundamental frequency is $0.53 \mathrm{~Hz}$. As expected, the vibration-development is not longer harmonic. Only the characteristic vibration of upper and lower section is in phase balance. Each time the lower end of the upper section hits the lower section, high frequent vibrations (e.g. 6.35Hz) are stimulated due to the impact. As seen in Movie 2, the acceleration signal of the high frequent vibrations shows an antiphase development.

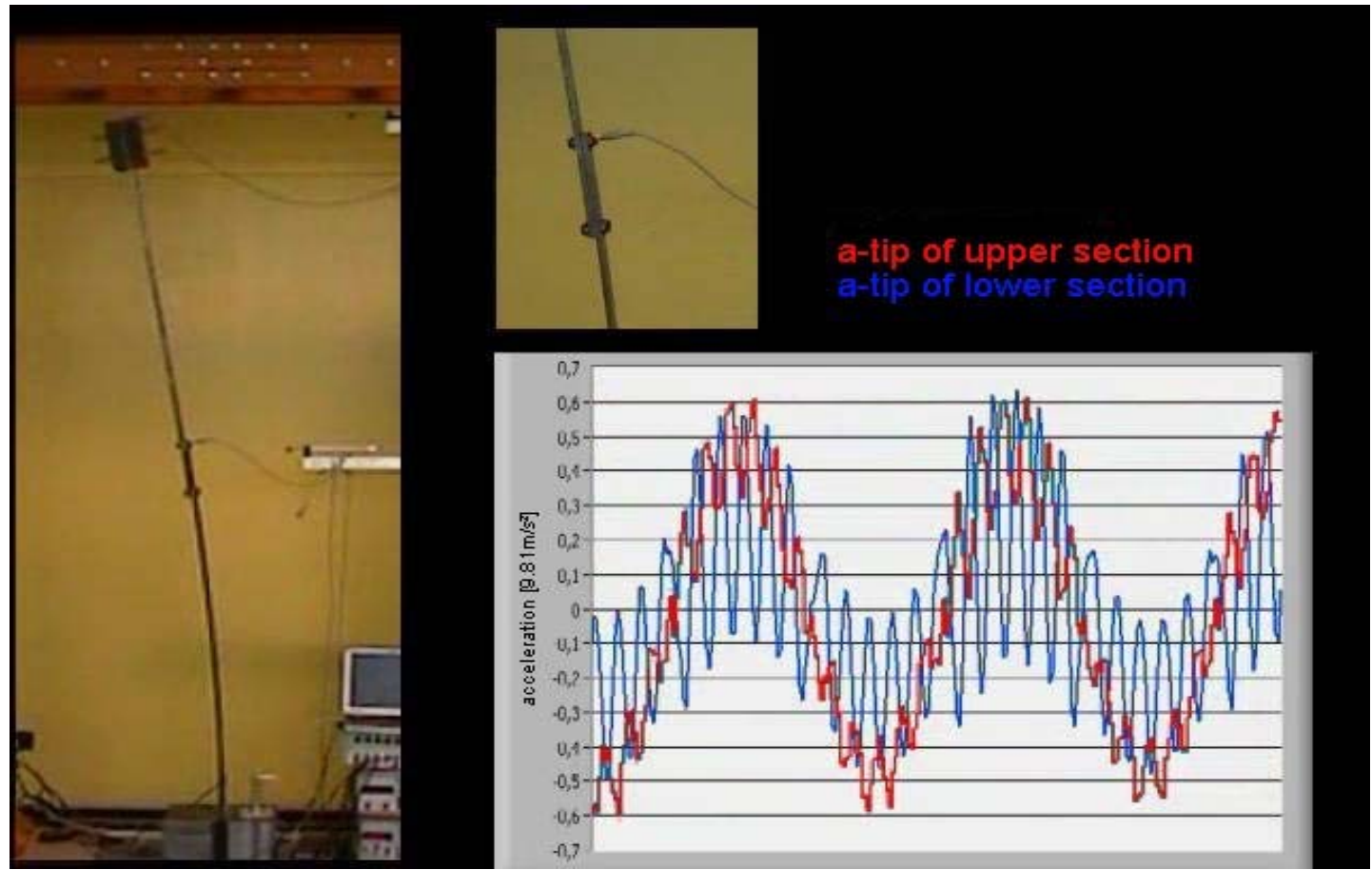

\section{Physical Modell}

From the viewpoint of mechanics, a non-linear multi-field problem of vibrating structural members with variable geometry has to be considered. Material surface areas of particular components move along surface areas of other components and define complicated boundary and transition conditions. The clearance produces non-linear effects. In many applications the different segments are slender and can be modelled as Bernoulli/Euler beams mounted on a rigid vehicle unit and carrying at some location, e.g., at the end of the last section, a load unit assumed to be rigid. The vehicle unit together with the first deformable segment and all the other segments (one of them together with the load) perform transverse motions and the extending or retracting motion of the sections is supplemented. The contact regions between two sections are modelled as discrete point contacts. A special feature of the modelling is to introduce the reaction forces at the contact points in the form of distributed line loads (by using Dirac impulse functions), so that for the contacting sections elementary boundary conditions remain. The contact formulation itself takes place via one-sided spring-damper elements.

The procedure is illustrated in Fig. 4a for a two-section telescopic beam system mounted on a rigid traverse, performing a translational motion accompanied by an extending motion of the two beam segments with defined clearance between them. Beam 1 is fixed at a rigid vehicle unit; beam 2 carries a point load at its end. The vehicle is driven by a horizontal force $F$ as excitation. The deformation of the beams (including vehicle mass and load) is represented by the absolute displacements $w\left(x_{1}, t\right)$ and $v\left(x_{2}, t\right)$. The model is defined by the following parameters: beam 
lengths $l_{1,2}$, constant cross-sectional areas $A_{1,2}$, constant cross-sectional moments of inertia $I_{1,2}$, density $\rho$ and Young's modulus $E$ of the two flexible components, masses of load and vehicle $m_{L}$ and $m_{T}$, respectively, and telescopic length $l_{A}(t)$. The contact between the beams is realised (see Figure $4 \mathrm{~b}$ ) via discrete spring-damper systems in the form of a so-called displacement condition (not a force condition) [3], the given number $n$ of contact points, the clearance $l_{S}$, spring stiffness $c$, and damping coefficient $d . c$ can be estimated from the geometry and the material of the contact partners whereas the estimation of $d$ is more complicated. As the purpose of the model is the creation of a control concept for vibration suppression, it is important that the equations of motion stay as simple as possible. In the controlled system the clearance plays the role of an external disturbance and as the controller has to work for every kind of contact, a very accurate estimation of $d$ is not necessary. In the axial direction it is assumed that there is no friction. This assumption is justifiable as the bearing between the different segments is realised as roller bearing in many applications. It is assumed here that the force flow leads from the upper part into the lower part.

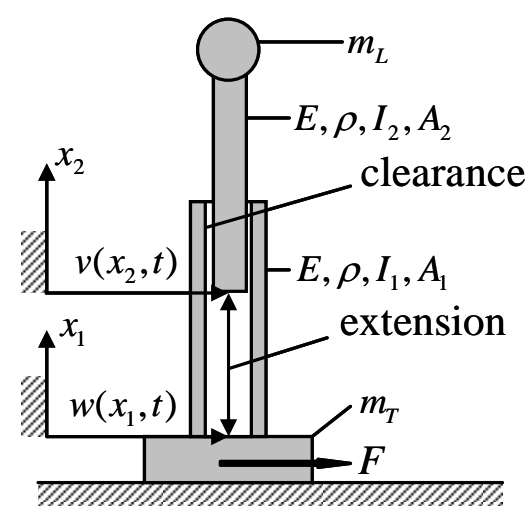

Fig. 4: a) System model

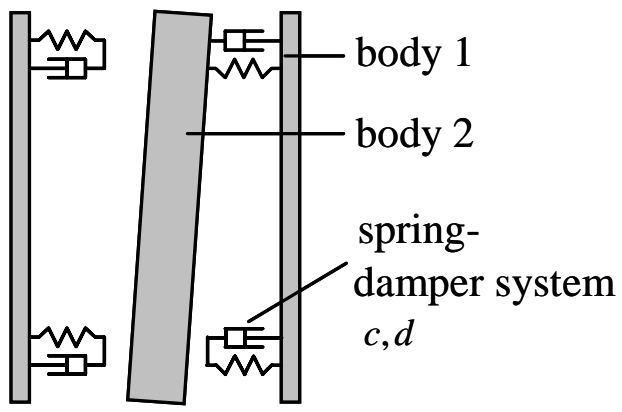

b) Contact formulation

\section{Formulation}

\section{Boundary value problem}

Applying Hamilton’s principle

$$
\delta \int_{t_{0}}^{t_{1}}(T-U) d t+\int_{t_{0}}^{t_{1}} W_{\text {virt }} d t=0
$$

the governing boundary value problem can be derived. $T$ is the kinetic energy, $U$ the potential energy and $W_{\text {virt }}$ the virtual work of forces without potential of the considered system. The kinetic energy reads

$$
T=\frac{1}{2} \int_{0}^{l_{1}} \rho A_{1}^{*} w_{t}^{2} d x_{1}+\frac{1}{2} \int_{0}^{l_{2}} \rho A_{2}^{*} v_{t}^{2} d x_{2}
$$

where $\rho A_{1}^{*}=\rho A_{1}+m_{T} \delta\left(x_{1}\right)$ and $\rho A_{2}^{*}=\rho A_{2}+m_{L} \delta\left(x_{2}-l_{2}\right)$ and the symbol $\delta($. represents Dirac's delta-function. If the action of the spring-damper systems is completely included into the virtual work, for the remaining potential energy one obtains 


$$
\begin{aligned}
U & =\frac{1}{2} \int_{0}^{l_{1}}\left[E I_{1} w_{x_{1} x_{1}}^{2}-\rho g\left(\int_{x_{1}}^{l_{1}} A_{1}^{*} d \bar{x}_{1}+\int_{0}^{l_{2}} A_{2}^{*} d x_{2}\right) w_{x_{1}}^{2}\right] d x_{1} \\
& +\frac{1}{2} \int_{0}^{l_{2}}\left[E I_{2} v_{x_{2} x_{2}}^{2}-\rho g \int_{x_{2}}^{l_{2}} A_{2}^{*} d \bar{x}_{2} v_{x_{2}}^{2}\right] d x_{2} .
\end{aligned}
$$

Since no internal damping of the beam segments will be taken into consideration, as the worst case for control, the virtual work contains all the contact forces between the beams and the locally concentrated driving force of the vehicle as distributed loads $f_{1}\left(x_{1}, t\right)$ and $f_{2}\left(x_{2}, t\right)$ which couple the resulting field equations:

$$
W_{\text {virt }}=\int_{0}^{l_{1}} f_{1} \delta w d x_{1}+\int_{0}^{l_{2}} f_{2} \delta v d x_{2} .
$$

Due to the formulation of all these locally concentrated forces by distributed loads using Dirac impulses, the boundary conditions will be homogeneous. Evaluating Hamilton's principle (2.1) introducing $T, U$ and $W_{\text {virt }}$ according to equations (2.2), (2.3) and (2.4), respectively, yields the governing field equations

$$
\begin{aligned}
\rho A_{1}^{*} w_{t t} & +E I_{1} w_{x_{1} x_{1} x_{1} x_{1}}+\rho A_{1} g\left[\left(l_{1}-x_{1}\right) w_{x_{1}}\right]_{x_{1}}+g\left(m_{L}+\rho A_{2} l_{2}\right) w_{x_{1} x_{1}} \\
& =f_{1}\left(x_{1}, t\right)+\delta\left(x_{1}-l_{1}\right) g\left(m_{L}+\rho A_{2} l_{2}\right) w_{x_{1}}, \\
\rho A_{2}^{*} v_{t t} & +E I_{2} v_{x_{2} x_{2} x_{2} x_{2}}+\rho A_{2} g\left[\left(l_{2}-x_{2}\right) v_{x_{2}}\right]_{x_{2}}+g m_{L} v_{x_{2} x_{2}} \\
& =f_{2}\left(x_{2}, t\right)-\delta\left(x_{2}\right) g\left(m_{L}+\rho A_{2} l_{2}\right) v_{x_{2}}+\delta\left(x_{2}-l_{2}\right) g m_{L} v_{x_{2}}
\end{aligned}
$$

and the corresponding boundary conditions

$$
\begin{aligned}
& w_{x_{1}}(0, t)=0, w_{x_{1} x_{1} x_{1}}(0, t)=0, w_{x_{1} x_{1}}\left(l_{1}, t\right)=0, w_{x_{1} x_{1} x_{1}}\left(l_{1}, t\right)=0, \\
& v_{x_{2} x_{2}}(0, t)=0, v_{x_{2} x_{2} x_{2}}(0, t)=0, v_{x_{2} x_{2}}\left(l_{2}, t\right)=0, v_{x_{2} x_{2} x_{2}}\left(l_{2}, t\right)=0
\end{aligned}
$$

for the two bodies.

For the special case in which the beam segments contact each other at the two points $x_{1}=l_{1}$ and $x_{2}=0$ only, the distributed forces are specified as

$$
\begin{aligned}
f_{1}= & \delta\left(x_{1}\right) F+\delta\left(x_{1}-l_{A}(t)\right)\left(F_{K}\left(\xi_{1}(t)\right)+\frac{d}{d t} \xi_{1}(t) \cdot D_{K}\left(\xi_{1}(t)\right)\right) \\
& +\delta\left(x_{1}-l_{1}\right)\left(F_{K}\left(\xi_{2}(t)\right)+\frac{d}{d t} \xi_{2}(t) \cdot D_{K}\left(\xi_{2}(t)\right)\right), \\
f_{2}= & -\delta\left(x_{2}\right)\left(F_{K}\left(\xi_{1}(t)\right)+\frac{d}{d t} \xi_{1}(t) \cdot D_{K}\left(\xi_{1}(t)\right)\right) \\
& -\delta\left(x_{2}-\left(l_{1}-l_{A}(t)\right)\right)\left(F_{K}\left(\xi_{2}(t)\right)+\frac{d}{d t} \xi_{2}(t) \cdot D_{K}\left(\xi_{2}(t)\right)\right) .
\end{aligned}
$$

The non-linear characteristic of the spring force $F_{K}(\xi(t))$ takes into account the fact that in the range of backlash no forces can be transferred. The same is valid for the assumed damping coefficient $D_{K}(\xi(t))$ : 


$$
\begin{gathered}
F_{K}(\xi(t))=c\left[\xi(t)-\frac{1}{2}\left(\xi(t)+\frac{l_{S}}{2}\right) \operatorname{sign}\left(\xi(t)+\frac{l_{S}}{2}\right)\right. \\
\left.+\frac{1}{2}\left(\xi(t)-\frac{l_{S}}{2}\right) \operatorname{sign}\left(\xi(t)-\frac{l_{S}}{2}\right)\right], \\
D_{K}(\xi(t))=d\left[1-\frac{1}{2} \operatorname{sign}\left(\xi(t)+\frac{l_{S}}{2}\right)+\frac{1}{2} \operatorname{sign}\left(\xi(t)-\frac{l_{S}}{2}\right)\right], \\
\xi_{1}(t)=v(0, t)-w\left(l_{A}(t), t\right), \xi_{2}(t)=v\left(\left(l_{1}-l_{A}(t)\right), t\right)-w\left(l_{1}, t\right) .
\end{gathered}
$$

\section{Discretisation}

The discretisation of the coupled partial differential equations (2.5) and (2.6) (nonlinear and timevariant in general) together with the corresponding boundary conditions (2.7) and (2.8) is based on Galerkin's method. For that, the approximate solutions $\bar{w}\left(x_{1}, t\right)$ and $\bar{v}\left(x_{2}, t\right)$ are represented by a series expansion

$$
\begin{gathered}
\bar{w}\left(x_{1}, t\right)=\sum_{i=1}^{N} u_{i}(t)\left(\cos \left(\lambda_{i} x_{1}\right)+\frac{\cos \left(\lambda_{i} l_{1}\right)}{\cosh \left(\lambda_{i} l_{1}\right)} \cosh \left(\lambda_{i} x_{1}\right)\right), \\
\bar{v}\left(x_{2}, t\right)=u_{N+1}(t)+u_{N+2}(t) x_{2}+\sum_{i=3}^{N} u_{N+i}(t)\left[\cosh \left(\kappa_{i} x_{2}\right)+\cos \left(\kappa_{i} x_{2}\right)\right. \\
\left.-\frac{\cosh \left(\kappa_{i} l_{2}\right)-\cos \left(\kappa_{i} l_{2}\right)}{\sinh \left(\kappa_{i} l_{2}\right)-\sin \left(\kappa_{i} l_{2}\right)}\left(\sinh \left(\kappa_{i} x_{2}\right)+\sin \left(\kappa_{i} x_{2}\right)\right)\right]
\end{gathered}
$$

fulfilling all boundary conditions (2.7) and (2.8).

Galerkin's averaging leads to a system of ordinary differential equations of the type

$$
\mathbf{M} \ddot{\mathbf{u}}=\mathbf{F}(\mathbf{u}, \dot{\mathbf{u}}, t) .
$$

\section{Vibration supression concept}

To suppress vibrations, a state space control concept is introduced. For a system without clearance and with a fixed telescopic length, equation (2.16) represents a time-invariant system of linear ordinary differential equations which can be reformulated as

$$
\mathbf{M u ̈}+\mathbf{C u}=\mathbf{b}^{*} F(t)
$$

where $\mathbf{b}^{*}$ is a $2 N$-dimensional vector, $\mathbf{M}$ is the mass matrix and $\mathbf{C}$ is the stiffness matrix of the system.

\section{Reduction of order}

Equation (3.1) represents a $2 N$-degree-of-freedom system. The objective of an order reduction is to find, for a given model of high order, a model of significantly lower order whose dynamic behaviour approximates the original behaviour as well as possible. This means that the approximate model has to contain the essential modes of the original system, since they dominate the dynamic behaviour of the original system [Lunze01]. For this purpose, the system equations (3.1) are transformed to principal coordinates $\mathbf{y}$ by 


$$
\mathbf{u}=\mathbf{M}_{R} \mathbf{y}
$$

The columns of the $(2 N, 2 N)$-matrix $\mathbf{M}_{R}$ will be composed of the right eigenvectors $\mathbf{m}_{R i}(i=1,2, \ldots, 2 N)$ of the system. $\mathbf{M}_{L}$ is a matrix which contains the left eigenvectors of the system $\left(\mathbf{M}_{L}^{T}=\mathbf{M}_{R}^{-1}\right)$. There is

$$
\mathbf{M}_{L}^{T} \mathbf{M}^{-1} \mathbf{C M}_{R}=-\operatorname{diag}\left(\omega_{1}^{2}, \omega_{2}^{2}, \ldots, \omega_{2 N}^{2}\right)=\mathbf{D}
$$

and $\omega_{i}^{2}(i=1,2, \ldots, 2 N)$ are the eigenvalues of the system.

From equation (3.1), using (3.2) and (3.3), it follows that

$$
\ddot{\mathbf{y}}=-\mathbf{D} \mathbf{y}+\mathbf{M}_{L}^{T} \mathbf{M}^{-1} \mathbf{b}^{*} F(t) .
$$

If then the eigenvectors corresponding to the large eigenvalues are removed from $\mathbf{M}_{R}$ and $\mathbf{M}_{L}$, one obtains the reduced $\left(2 N, N_{r}\right)$-matrices $\mathbf{M}_{R r}$ and $\mathbf{M}_{L r}$, and with that the approximate model is

$$
\ddot{\mathbf{y}}_{r}=-\mathbf{D}_{r} \mathbf{y}_{r}+\mathbf{M}_{L r}^{T} \mathbf{M}^{-1} \mathbf{b}^{*} F(t) .
$$

This type of order reduction is justified since the state control should control the rigid body motion and the lower modal vibrations of the system. The high-frequency oscillations possess a small magnitude and will diminish strongly by material damping effects. The number of higher-order modes to be included into the reduced model has to be determined depending on the application and the quality of control desired.

\section{Driving unit}

The driving unit of the telescope will be represented by a scalar system of first order

$$
T_{A} \dot{F}+F=K_{A} U
$$

with time constant $T_{A}$ and amplification factor $K_{A}$. $U$ is the control voltage of the motor. Introducing the state variables

$$
z_{1}=y_{r 1}, \ldots, z_{N_{r}}=y_{r N_{r}}, z_{N_{r}+1}=\dot{y}_{r 1}, \ldots, z_{2 N_{r}}=\dot{y}_{r N_{r}}, z_{2 N_{r}+1}=F,
$$

instead of (3.5) and (3.6) one obtains

$$
\dot{\mathbf{z}}=\mathbf{A z}+\mathbf{b} U
$$

\section{Controllability and observability}

Controllability and observability are checked by computing the so-called controllability matrix $\mathbf{Q}_{S}$ and observability matrix $\mathbf{Q}_{B}$ [Föllinger78]:

$$
\begin{gathered}
\mathbf{Q}_{S}=\left[\mathbf{q}_{S 1}, \mathbf{q}_{S 2}, \ldots, \mathbf{q}_{S N^{*}}\right], \mathbf{q}_{S 1}=\mathbf{b}, \mathbf{q}_{S i+1}=\mathbf{A} \mathbf{q}_{S i}, \\
\mathbf{Q}_{B}=\left[\mathbf{q}_{B 1}, \mathbf{q}_{B 2}, \ldots, \mathbf{q}_{B N^{*}}\right]^{T}, \mathbf{q}_{B 1}=\mathbf{c}_{w F}, \mathbf{q}_{B i+1}=\mathbf{A}^{T} \mathbf{q}_{B i}
\end{gathered}
$$


where

$$
\begin{gathered}
N^{*}=2 N_{r}+1 \\
\mathbf{c}_{w F}=\left[\left[W_{1}(0), W_{2}(0), \ldots, W_{N}(0), 0, \ldots, 0\right] \mathbf{M}_{R r} \mathbf{S}\right]^{T}
\end{gathered}
$$

and $\mathbf{S}$ comes from

$$
\mathbf{y}_{r}=\mathbf{S z}
$$

The system is completely controllable if the determinant of $\mathbf{Q}_{S}$ does not vanish, and it is completely observable if the determinant of $\mathbf{Q}_{B}$ does not vanish. For real systems, both conditions are usually fulfilled, but it should be mentioned that for a very large mass $m_{T}$ problems with the observability may occur since then the reaction of the telescope vibrations on the motion of the base is very weak.

\section{Control design by pole placement}

The control synthesis in the state space is directed to the goal of taking the state of the system from an initial state $\mathbf{z}_{0}$ to the state $\mathbf{z}_{E}=0$ fulfilling demands on the dynamic behaviour of the system. The poles of the feedback control loop determine the transfer behaviour, and therefore they have to be selected in such a manner that the requirements on the dynamic behaviour are fulfilled. This leads to the desired characteristic polynomial of the closed control loop:

$$
p(s)=p_{0}+p_{1} s+\ldots+p_{N^{*}-1} s^{N^{*}-1}+s^{N^{*}}
$$

To achieve this aim, the $N^{*}$-dimensional vector $\mathbf{r}$ (see Figure 2) as defined by $J$. Ackermann reads [Föllinger78].

$$
\mathbf{r}^{T}=p_{0} \mathbf{q}_{S}^{T}+\ldots+p_{N^{*}-1} \mathbf{q}_{S}^{T} \mathbf{A}^{N^{*}-1}+\mathbf{q}_{S}^{T} \mathbf{A}^{N^{*}}
$$

where $\mathbf{q}_{S}^{T}$ is the last row of the inverse controllability matrix $\mathbf{Q}_{S}^{-1}$ (see (3.9)).

For a constant or a slowly changing (compared to the control loop) command variable $w_{F S}$ (see Figure 5), the pre-filter $S$ (see Figure 5 and [Föllinger78], for instance) reads

$$
S=\frac{1}{\mathbf{c}_{w F}^{T}\left(\mathbf{b} \mathbf{r}^{T}-\mathbf{A}\right)^{-1} \mathbf{b}}
$$




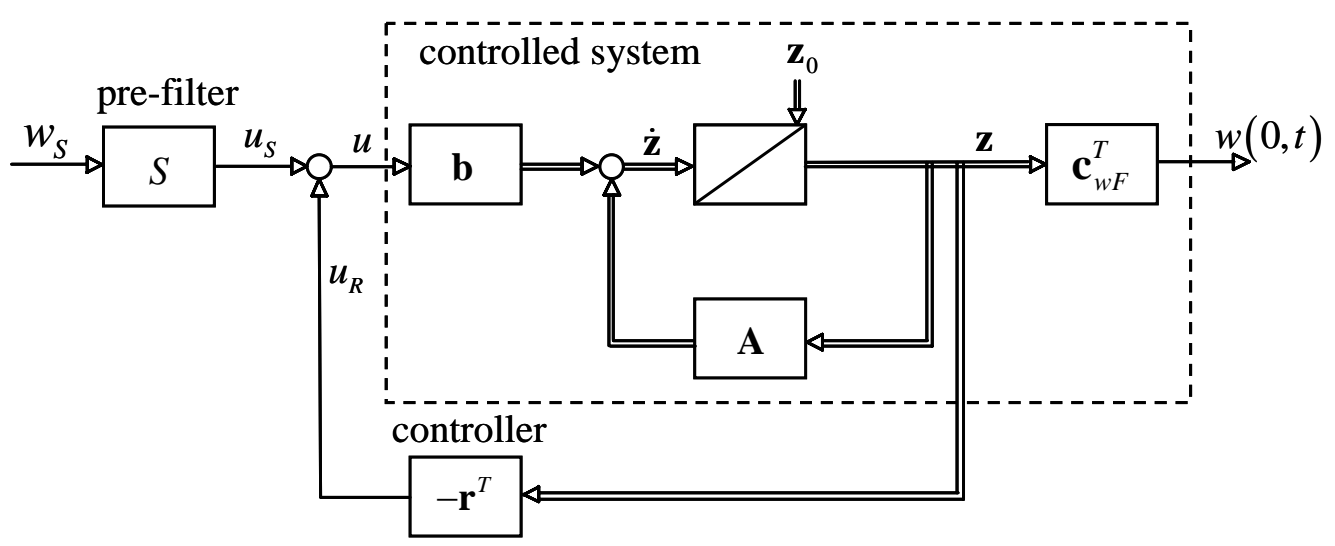

Fig. 5: State space control loop.

\section{Luenberger observer}

For the present problem, the state vector $\mathbf{z}$ cannot be measured directly, but the control voltage $U$ of the motor and the displacement $w(0, t)$ of the base are measurable. The objective of the so-called Luenberger observer is to find from this information an approximate value $\hat{\mathbf{z}}$ of $\mathbf{z}$. The observer then is written as

$$
\dot{\hat{\mathbf{z}}}=\mathbf{F} \hat{\mathbf{z}}+\mathbf{b} U+\mathbf{k} w(0, t) .
$$

The eigenvalues of $\mathbf{F}$ are prescribed and placed on the left of the eigenvalues of the closed control loop, which leads to the desired characteristic polynomial

$$
f(s)=f_{0}+f_{1} s+\ldots+f_{N^{*}-1} s^{N^{*}-1}+s^{N^{*}}
$$

According to (3.15) $\mathbf{k}$ then reads

$$
\mathbf{k}=f_{0} \mathbf{q}_{B}+\ldots+f_{N^{*}-1} \mathbf{A}^{N^{*}-1} \mathbf{q}_{B}+\mathbf{A}^{N^{*}} \mathbf{q}_{B}
$$

where $\mathbf{q}_{\mathbf{B}}$ is the last column of the inverse observability matrix $\mathbf{Q}_{B}^{-1}$ (see (3.10)).

F reads [Föllinger78]

$$
\mathbf{F}=\mathbf{A}-\mathbf{k c}_{w F}^{T} .
$$

\section{Telescopic operations with clearance}

For real telescopic operations the parameters of the controller and of the observer are determined for different telescopic lengths and approximated by polynomials which leads to an adaptive controller $\mathbf{r}\left(l_{A}(t)\right), S\left(l_{A}(t)\right)$ and observer $\mathbf{F}\left(l_{A}(t)\right), \mathbf{b}\left(l_{A}(t)\right), \mathbf{k}\left(l_{A}(t)\right)$. Due to the Luenberger observer, straightforward measurements of the motion of the telescope base and of the control variable of the actuator are sufficient to operate the controller. This makes it possible, to apply the controller, developed for the reduced linear system model, to the significantly more complicated system with clearance (2.16) for studying the influence of clearance on the vibration suppression during telescopic motions. 


\section{Simulation Results}

Quantitative results are presented here for a 2-sectional system. The results should illustrate the effect of the controller on a telescopic system with and without clearance and only represent a small extract of the existing results. For better comparison of the measured dynamic behaviour and the simulation results, the parameters are taken originally from the above described and illustrated test rig:

$$
\begin{aligned}
& l_{1}=l_{2}=1.35 \mathrm{~m}, A_{1}=A_{2}=0.001 \mathrm{~m}^{2}, I_{1}=I_{2}=0.83 \cdot 10^{-8} \mathrm{~m}^{4}, \rho=7850 \mathrm{~kg} / \mathrm{m}^{3}, \\
& m_{T}=100 \mathrm{~kg} m_{L}=17.897 \mathrm{~kg}, E=2.1 \cdot 10^{11} \mathrm{~N} / \mathrm{m}^{2}, c=10^{7} \mathrm{~N} / \mathrm{m}, d=10^{3} \mathrm{Ns} / \mathrm{m}, n=3 .
\end{aligned}
$$

The calculation results are based on 4-term truncations (2.14) and (2.15). The controller influences the rigid body motion and the first modal vibration of the system. The poles of the closed control loop and of the Luenberger observer are placed on the points -8 and -12 in the complex plane.

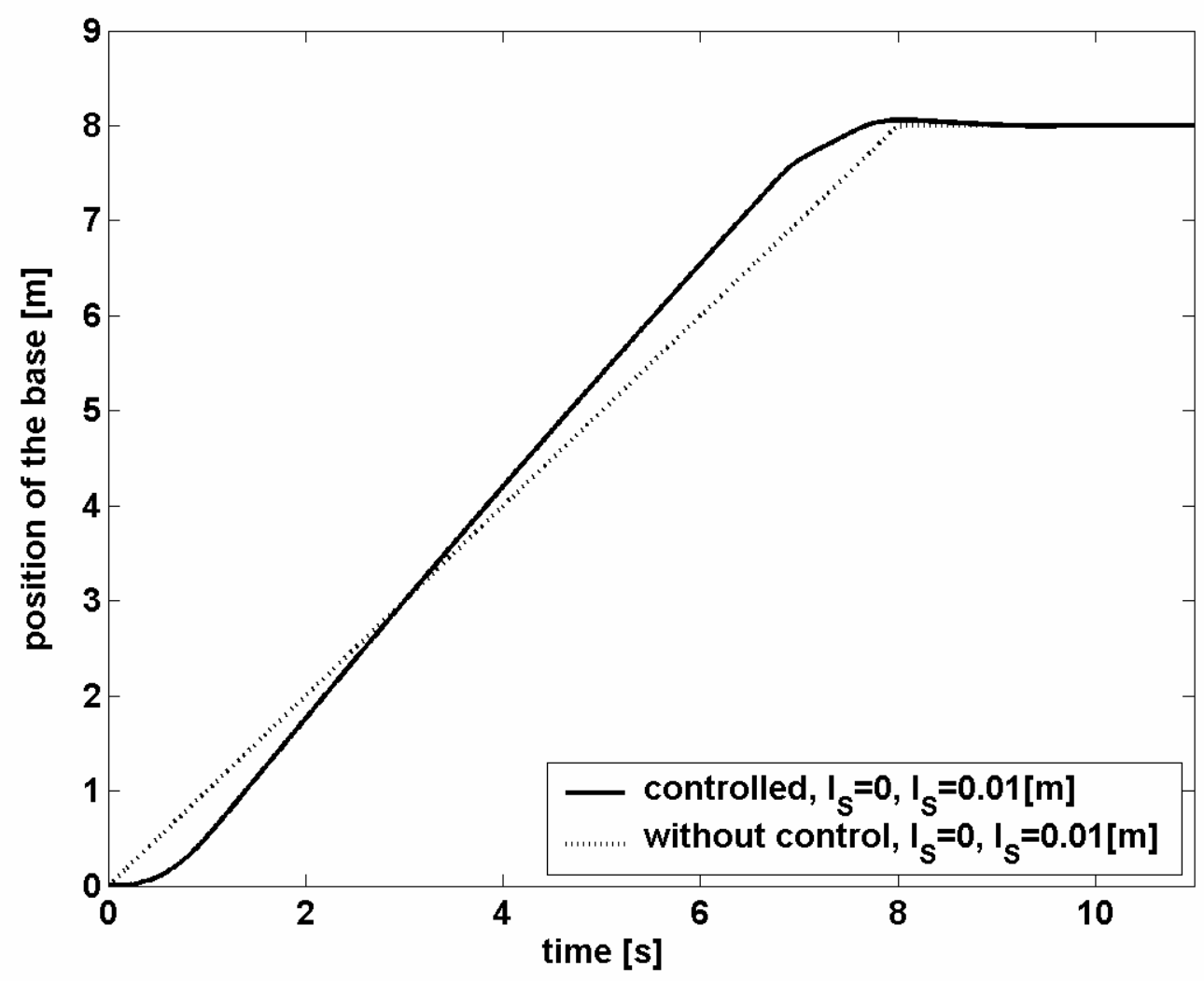

Fig. 6: Position of the base.

The system starts from an initial point without any initial velocity and has to cover a straight distance of $8 \mathrm{~m}$ before it stops after $8^{\mathrm{S}}$. Figure 6 shows the position of the base of the telescope versus time for a prescribed velocity of $1 \mathrm{~m} / \mathrm{s}$ and for a motion prescribed by the controller. Both simulations are done with $\left(l_{s}=0.01 \mathrm{~m}\right)$ and without $\left(l_{s}=0 \mathrm{~m}\right)$ clearance. During the simulations, the telescopic length increases from $l_{A}(0)=0.15 m$ to $l_{A}(8)=1 m$ with constant velocity. Figure 7 shows the position of the telescope tip relative to its base during the motion and illustrates the vibration suppression by state control. The remaining deflection of the relative position in the simulation with clearance comes from the tilted position of the upper segment in the lower segment due to clearance. 


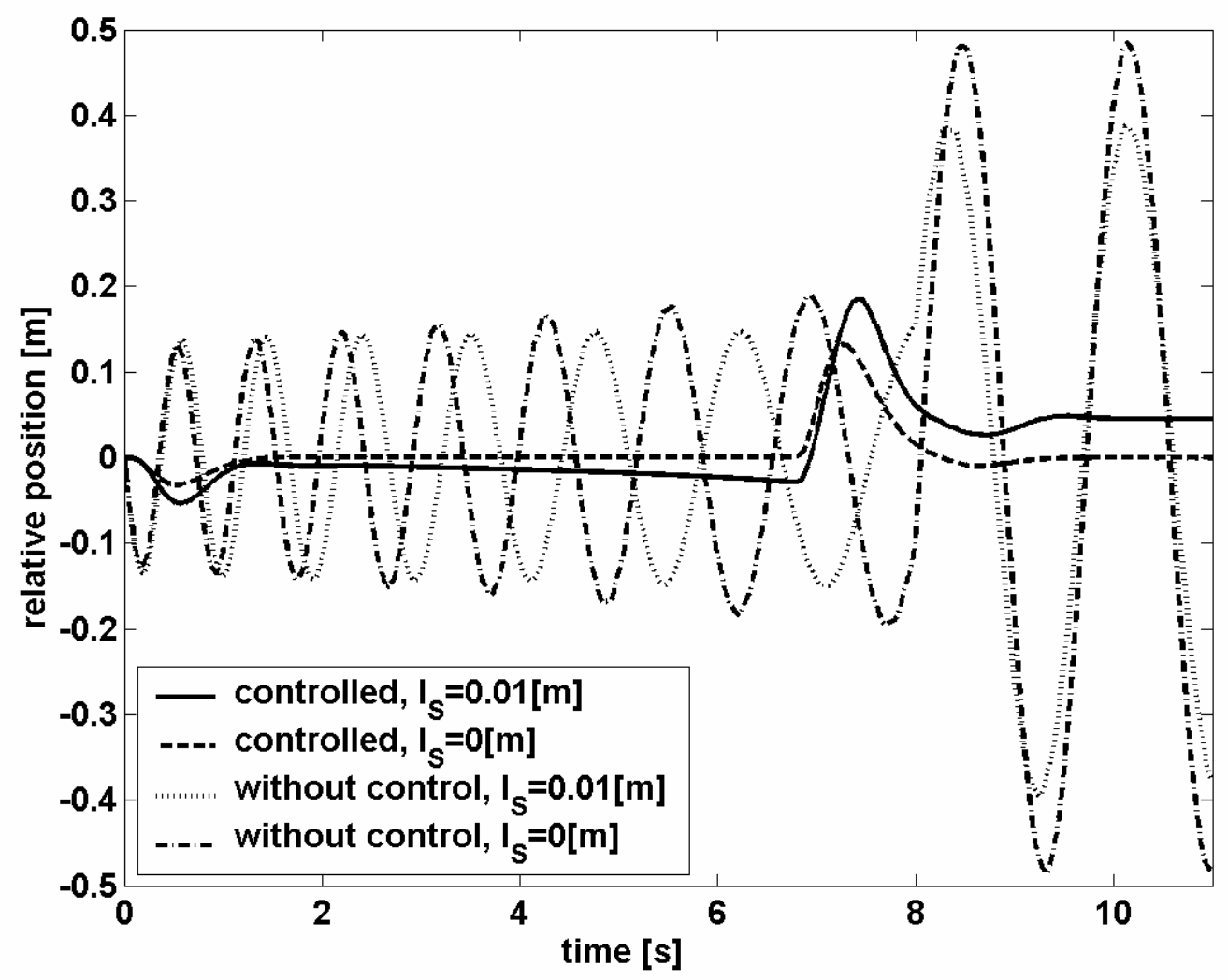

Fig. 7: Relative position.

Movie 3a) (controlled) and Movie 3b) (without control) show animations of the computational results with clearance. The red lines represent the lower segment, the blue line represents the upper segment, as seen from the point of view of an observer moving with the base of the telescope. 


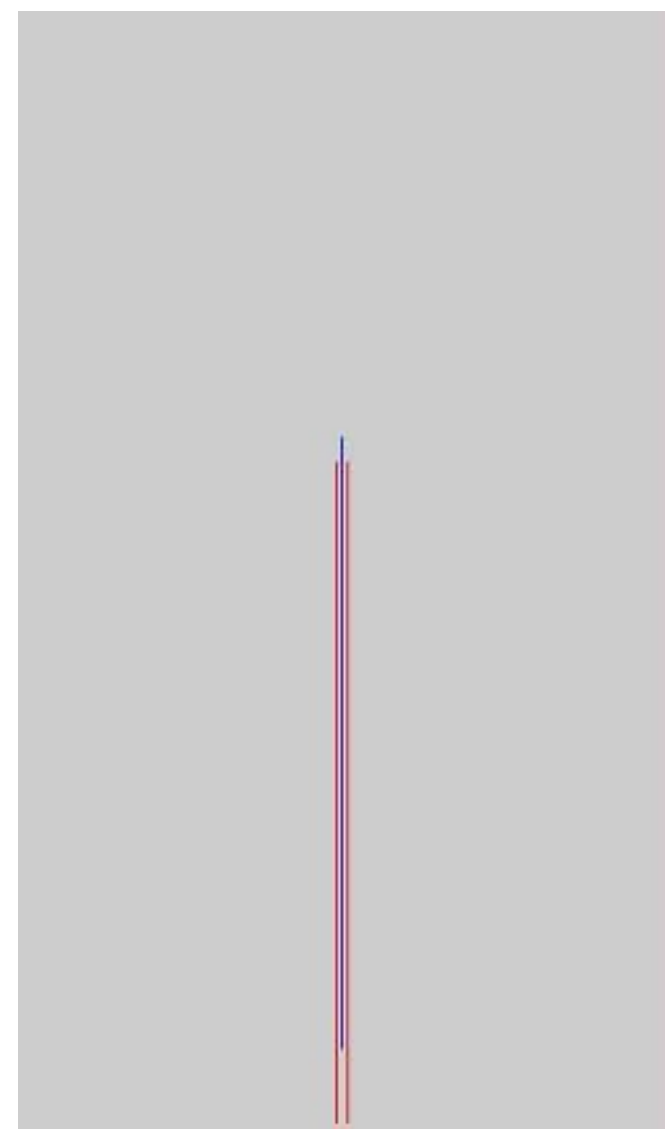

Mov. 3a): controlled

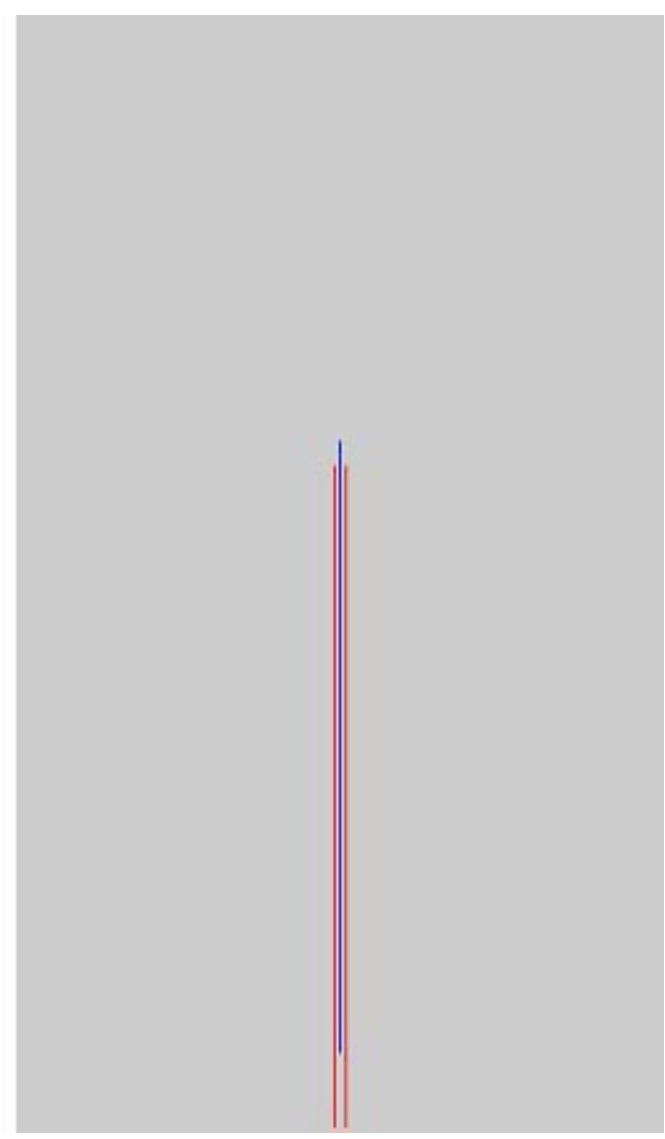

Mov. 3b): uncontrolled

\section{Conclusions}

To improve efficiency and to overcome possible safety problems of multi-section constructions during acceleration and deceleration in driving direction and additional extending and retracting motion of the sections, a vibration suppression in such structural systems of variable geometry seems to be useful. To achieve this, an appropriate modelling of the system together with the development of an efficient control strategy are the essential problems to be treated. For slender beam-shaped structural members, the present contribution has suggested an approach to find a good solution with a justifiable computational expense.

To suppress unavoidable vibrations, the concept of state control via pole placement seems to be very efficient. Based on a model reduction, it is possible to design a control approach which exhibits the desired effects without extensive effort. Introducing a so-called Luenberger observer, straightforward measurements of the motion of the telescope base and of the control variable of the actuator are sufficient to operate the controller. Additionally, this makes it possible to apply the controller developed for the reduced linear system model to the significantly more complicated system with clearance for studying the influence of clearance on the closed control loop. The straightforward handling opens the way for exhaustive parameter studies. To evaluate the results, the existing test rig will be modified and used further on.

\section{Literature}

[Barthels05] Barthels, P.; Wauer, J.: Modeling and Dynamic Analysis of Telescopic Systems of Structural Members with Clearance. IN: Nonlinear Dynamics 42(2005)4, S. 371-382, DOI: 10.1007/s11071-005-6788-7

[Föllinger78] Föllinger, O.: Regelungstechnik. Berlin: Elitera 1978 
[Fujita03] Fujita, Y.; Shiona, K.; Koike, K.; Koseki, T.: Multibody Dynamics of the 2 Section Ladder Model Extending and Retracting Each Other. Proceedings of ASME-DETC’ 03, Chicago (USA), Sept. 2-6, 2003, VIB-48367.

[Lunze01] ～Lunze, J.: Regelungstechnik 1. Berlin [u.a.]: Springer 2001.

[Waltersberger04] Waltersberger, B.; Keppler, R.; Wauer, J.: Stoßsimulation mittels einseitiger Feder-Dämpfer Kontakte. IN: PAMM - Proceedings in Applied Mathematics and Mechanics 4(2004)1, S. 119-120. DOI: 10.1002/pamm.200410041

Volltext: http://www3.interscience.wiley.com/cgi-bin/fulltext/

109800497/PDFSTART 